Evidence Updates

\title{
Yoga and Naturopathy intervention for reducing anxiety and depression of Covid-19 patients - A pilot study
}

\author{
R. Jenefer Jerrin ${ }^{\mathrm{a}}$, S. Theebika ${ }^{\mathrm{b}}$, P. Panneerselvam ${ }^{\mathrm{c}}$, ST. Venkateswaran ${ }^{\mathrm{b}}$, N. Manavalan ${ }^{\mathrm{a}}$, \\ K. Maheshkumar ${ }^{\mathrm{d}, "}$ \\ ${ }^{a}$ Department of Naturopathy, Government Yoga and Naturopathy Medical College and Hospital, Chennai, India \\ ${ }^{\mathrm{b}}$ Department of Yoga, Government Yoga and Naturopathy Medical College and Hospital, Chennai, India \\ ${ }^{\mathrm{c}}$ Department of Physiology, Government Erode Medical College, Perundurai, Erode, Tamilnadu, India \\ d Department of Physiology and Biochemistry, Government Yoga and Naturopathy Medical College and Hospital, Chennai, India
}

\section{A R T I C L E I N F O}

\section{Keywords:}

Covid-19

Anxiety

Depression

Mental health

Yoga

Naturopathy

\begin{abstract}
A B S T R A C T
Background: Coronavirus disease 2019 (Covid-19) infection has caused a prominent psychological and mental havoc among the patients. Till now, there is paucity in literature for the management of psychological problems among patients with Covid-19. So we aimed in our study to assess the effect of Yoga and Naturopathy intervention on anxiety and depression of Covid-19 patients.

Methods: This quasi experimental study was conducted among 130 Covid-19 positive patients admitted in a tertiary care hospital. Yoga and Naturopathic intervention was given for $60 \mathrm{~min}$ a day for two weeks. Hospital anxiety depression scale (HADS) and Corona anxiety scale (CAS) was used to assess the generalized anxiety and depression among the patients.

Results: Average age of the patients participated was 44 with the range from 36 to 53 years. Among them 85 were male and 45 were female. Of all 130 patients, 33\% had symptoms of borderline depression, 9.2\% had severe depression, $40 \%$ had borderline anxiety and $12.3 \%$ had severe anxiety. In CAS, 59 patients (45.38\%) reported Covid-19 related dysfunctional anxiety. All the patients showed a significant reduction in the HADS- anxiety (P $<0.01$ ), HADS-depression ( $\mathrm{p}<0.01$ ) and CAS ( $\mathrm{p}<0.01$ ) score after the intervention.

Conclusion: The present study showed significant reduction of anxiety and depression level among the Covid-19 patients. These interventions can be added to the conventional care for better mental and physical wellbeing of the patients after validating the findings with justified study design.
\end{abstract}

\section{Background}

On December 31, 2019, China reported a cluster of pneumonia (Covid-19) cases of unknown cause that later identified as severe acute respiratory syndrome coronavirus 2 (SARS-CoV-2). ${ }^{1}$ Patients with Covid-19 infection presented fever, cough, and shortness of breath within 2-14 days after exposure. ${ }^{2},{ }^{3}$ In recognition of the widespread global transmission of Covid-19, the World Health Organization (WHO) declared Covid-19 to be a pandemic on March 11, 2020. ${ }^{4}$ In India, Covid-19 pandemic was managed very effectively with nationwide lockdown from March 24, 2020. Till date more than 12 million people have been infected with SARS-CoV-2 virus and more than 0.162 million deaths reported in India. ${ }^{5}$ This pandemic crisis was efficiently managed by Government of India (GoI), with the direction of WHO and Indian council of Medical research (ICMR) guidelines. AYUSH ministry also has taken many initiatives for the control of Covid-19 pandemic and recommended various complementary and alternative (CAM) therapies as a supportive and adjuvant treatment. ${ }^{6,7}$ In Tamilnadu, the state government along with directorate of Indian Medicine has approached with constructive mode and recommended an advisory on implementing Yoga \& Naturopathy treatments together with allopathic management for Covid-19 patients across the state in all COVID care centers. The aim of the intervention was to improve their physical and mental health in addition to that of conventional care for the Covid-19 infection. The previous findings from the systematic review and meta-analysis report that the prevalence of psychological morbidities were high among

\footnotetext{
* Corresponding author. Department of Physiology and Biochemistry, Assistant Medical officer/Lecturer Grade II, Government Yoga and Naturopathy Medical college and Hospital, Chennai, 600106, India.

E-mail address: doctor.mahesh1985@gmail.com (K. Maheshkumar).
} 
Covid-19 patients. $^{8}$ They have found that about half of the population faced psychological impacts such as poor sleep quality (40\%), stress (34\%), and psychological distress (34\%). An Indian survey conducted among the common people during the Covid-19 lockdown has reported that $74 \%$ had a moderate level of stress, $40 \%$ stated anxiety/depressive symptoms. ${ }^{9}$ However there was a lacuna in the studies on the psychological profile of the Covid-19 patients and its management. So the present study aimed to assess the effect of Yoga and Naturopathy interventions on anxiety and depression level among Covid-19 positive patients.

\section{Methods}

\subsection{Participants}

This quasi experimental study was conducted on Covid-19 positive patients confirmed by RT-PCR of naso-phayngeal swab for SARS CoV-2 at tertiary care hospital, Erode, Tamilnadu. A total of 130 patients (since it's a pilot study sample size estimation was not done) with the age group 20-65 years were recruited. The written informed consent was obtained from the patients and Institutional ethics permission also obtained for the study [IEC/001/GEMC\& H/2020]. Inclusion criteria were Covid-19 positive patients of both sex, asymptomatic or with mild to moderate symptoms, and willing to participate in the study. Exclusion criteria were patients with metabolic disorders (diabetes, hypertension, and obesity), need oxygen supply or ventilation support and pregnant women.

\subsection{Intervention}

Yoga and Naturopathic intervention was given for $60 \mathrm{~min}$ a day for two weeks in the morning between 7.30am and 8.30am. The details of the interventions are presented in table:1. Qualified Yoga and Naturopathy doctors gave the intervention with personal protective equipment (PPE) kits during the intervention. In addition, this intervention was also shared to the patients in the form of an $\mathrm{E}$ module (video) for better self-learning and practice. A log book was maintained to note the timing and duration of practice per day to ensure that the entire study group practiced consistently. This study was executed between the month of June and October 2020.

\subsection{Outcome measurement}

HADS (Hospital anxiety depression scale) and CAS (Corona anxiety scale) score was used to assess the generalized anxiety and depression among the participants. The HADS consists of 14 items, seven items for the anxiety and seven for the depression. Each item was rated on a 4point (0-3) scale and a score between 0 and 7 is "normal," between 8 and 10 "borderline," and greater than 11 "severe". ${ }^{10}$ CAS is a self-report mental health questionnaire for anxiety associated with the Covid-19 crisis. It has five items and each item rated on a 5-point scale (from

Table: 1

Yoga and Naturopathy interventions details.

\begin{tabular}{lll}
\hline Therapies & Sessions & Duration \\
\hline Yoga & 2 session/day & $15 \mathrm{~min}$ \\
Tadasana & 5 rounds & \\
Ardha kati chakrasana & 5 rounds & \\
Ardha chakrasana & 5 rounds & $20 \mathrm{~min}$ \\
Pranayama & 2 session/day & \\
Hand stretch & 20 cycle & \\
Hand in and out & 20 cycle & \\
Nadi shuddhi & 20 cycle & \\
Bhramari & 20 cycle & $5 \mathrm{~min}$ \\
Steam inhalation & 2 session/day & $5 \mathrm{~min}$ \\
Salt water gargling & 2 session/day & $15 \mathrm{~min}$ \\
Heliotherapy (sun exposure) & 2 session/day & \\
\hline
\end{tabular}

0 -not at all to 4 -nearly every day) based on past two weeks experiences. A total score $\geq 9$ shows possible dysfunctional Covid-19 related anxiety. ${ }^{11}$ Questionnaires were administered before and after the intervention by co-researchers who wre not involved in the study.

\subsection{Statistical analysis}

The descriptive analysis was conducted for the questionnaires used in the study. Cronbach's alpha was computed for the internal reliability scores for each scale. Confirmatory factor analysis was performed to confirm the factor structure of the scales used for this study. Statistical analysis was performed using R statistical software version 4.0.2 for Windows and data expressed in Mean with standard deviation (SD). Normality of data was tested using Kolmogrov-Smirnov test. Paired $t$ test was performed to test the statistical significance for intervention. Statistical significance was accepted at $\mathrm{P}<00.05$.

\section{Results}

All the extracted factors of the scales had good internal consistency of $\geq 0.9$. Confirmatory factor analysis (CFA) also showed that most of the fit statistics for all the scales were in the acceptable range (Table: 2 ). Descriptive characteristic for the patients are presented in table: 3 . Out of 130 patients, 85 male and 45 female with the average age of 44 years participated in the study. All the recruited patients successfully completed the study without any adverse events. Most of the patient had travel history (n-120) and minimum stay in the hospital was 4 days. Of all 130 patients, $33 \%$ had symptoms of borderline depression, $9.2 \%$ had severe depression, $40 \%$ had borderline anxiety and $12.3 \%$ had severe anxiety. In CAS, 59 patients (45.38\%) reported Covid-19 related dysfunctional anxiety. All the patients showed a significant reduction in the HADS- anxiety ( $P<0.05$, Figure: 1$)$, HADS-depression $(\mathrm{p}<0.04$, Figure: 2 ) and CAS ( $<0.01)$ score after the intervention (Figure: 3 ).

\section{Discussion}

The findings demonstrate that patients have reported a reduction in anxiety and depression level after the Yoga and Naturopathy interventions. We found that $45 \%$ of the patients experienced Covid-19 related anxiety and it was similar to that of the previous finding. ${ }^{12,13}$ Another recent study supported our findings and showed that yoga practice is an effective self-management approach to cope with stress, anxiety and depression during Covid-19 lockdown. ${ }^{14,15}$ In addition, many studies have been done previously with various forms of yoga practices for the mental and physical benefits. ${ }^{16-18}$ Regular practice of yoga and other natural therapies have shown to promote and improve the respiratory and cardiovascular functions. ${ }^{6}$ The improved physiological functions in turn are believed to reduce the stress, anxiety, depression, and enhance overall well-being of the practitioners. ${ }^{15}$

Table 2

Goodness of fit statistics for confirmatory factor analysis.

\begin{tabular}{llll}
\hline Variables of Goodness of fit & Reference value & HASD & CAS \\
\hline$\chi 2$ (df) & - & $345.6(76)$ & $43.82(5)$ \\
$\chi 2 /$ df & $<5$ & 4.53 & 8.7 \\
P value & $>0.05$ & 0.00 & 0.00 \\
RMSEA & $<0.08$ & 0.06 & 0.24 \\
CFI & $>0.90$ & 0.50 & 0.93 \\
GFI & $>0.90$ & 0.89 & 0.88 \\
AGFI & $>0.90$ & 0.86 & 0.64 \\
SRMR & $<0.08$ & 0.089 & 0.04 \\
NFI & $>0.90$ & 0.86 & 0.92 \\
\hline
\end{tabular}

$\chi 2$; Model Chi Square, df; degrees of freedom, GFI; Goodness-of-Fit Index, AGFI; Adjusted Goodness of Fit, NFI; Normed Fit Index, CFI; Comparative fit index, RMSEA; root mean square error of approximation, SRMR; standardized root mean square residual. 
Table: 3

Details of the patients participated in the study.

\begin{tabular}{ll}
\hline Variables & Details \\
\hline Age (yr) & $44.32 \pm 12.09$ [36-53] \\
Sex (M/F) & $85 / 45$ \\
Height (cm) & $162.53 \pm 11.80$ \\
Weight (Kg) & $69.74 \pm 11.05$ \\
Married (Yes/No) & $112 / 18$ \\
Smoking (Yes/No) & $13 / 117$ \\
Alcohol intake (Yes/No) & $10 / 120$ \\
Occupation & \\
Government & 17 \\
Private & 38 \\
Self & 39 \\
Unemployment & 36 \\
Positive patients in Family (Yes/no) & $63 / 67$ \\
Travel history (Yes/No) & $120 / 10$ \\
Length of Stay in days & $4 \pm 3[2-7]$ \\
HADS: Depression - borderline/severe case & $43(33 \%) / 12(9.2 \%)$ \\
HADS: Anxiety - borderline/severe case & $52(40 \%) / 16(12.3 \%)$ \\
CAS $\geq 9$ (dysfunctional anxiety) & $59(45.38)$ \\
\hline
\end{tabular}

\section{HADS - Anxiety Score}

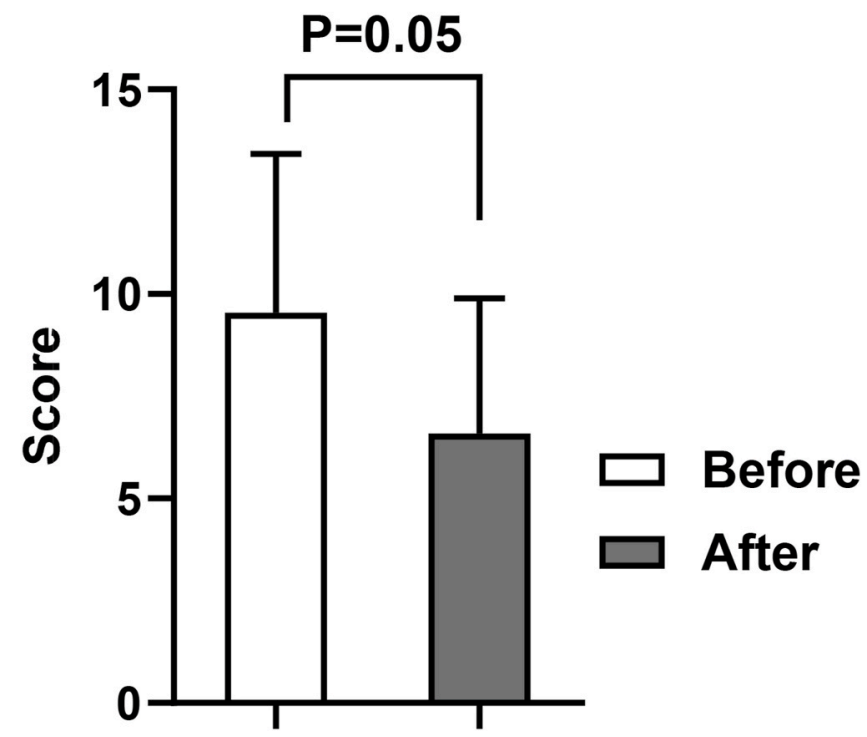

Fig. 1. HADS Anxiety Score before and after intervention.

Interestingly, patients who showed severe depression and anxiety score before intervention reported the scores within the normal limits after intervention. Perhaps the routine practice of yoga with the guidance of experienced doctors have helped the patients to calm their mind and maintain a positive personality during the Covid-19 treatment in the isolation ward. This finding further paves way to the point that yoga and naturopathy interventions could be used as a complementary and alternative therapy to manage the psychological problems for Covid-19 patients. To our knowledge, current study is the first to show variations in the psychological profile following Yoga and Naturopathy intervention for Covid -19 patients. Limitations of our study are not having a control group and being a non-randomized trial with limited sample size as it is a preliminary study. Still further studies with justified study design have to be done for validation of the outcomes.

\section{Conclusion}

Yoga and Naturopathy interventions significantly reduced the

\section{HADS - Depression Score}

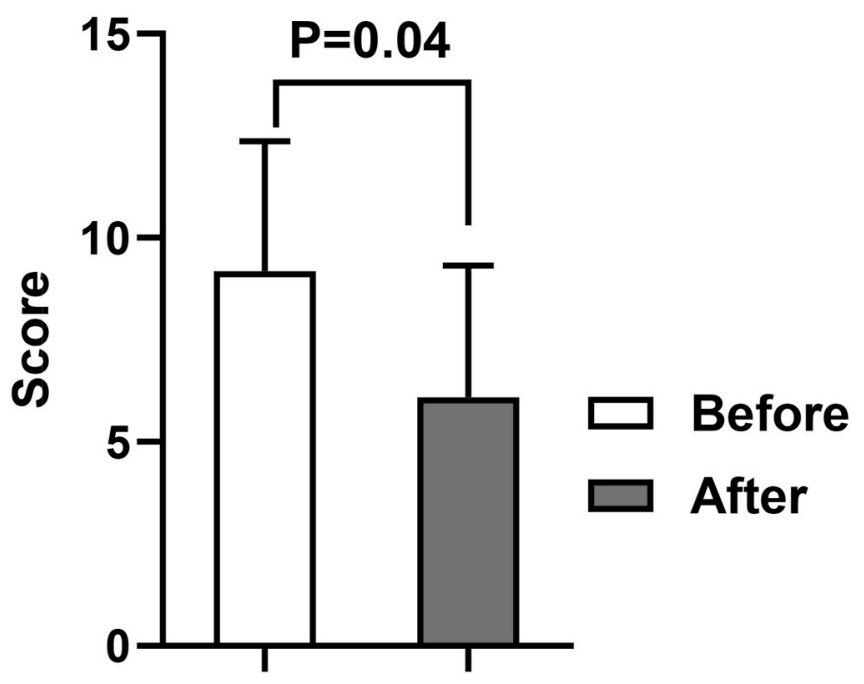

Fig. 2. HADS- Depression Score before and after intervention.

\section{Corona Anxiety scale (CAS)}

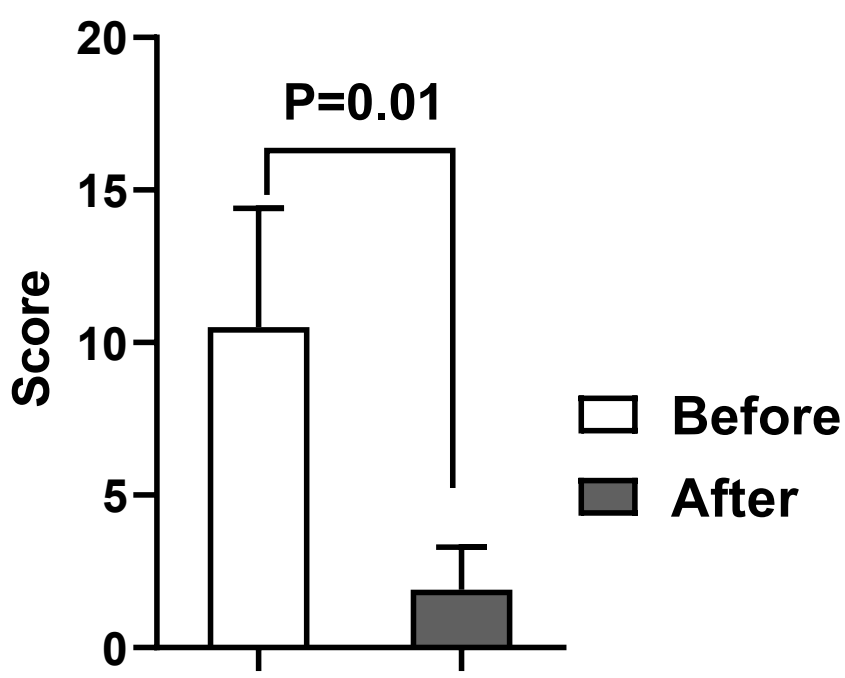

Fig. 3. Corona Anxiety Scale (CAS) before and after intervention.

anxiety and depression among the patients with covid-19 infection. These interventions can be added to the conventional care for better mental and physical wellbeing to the patients.

Declaration of competing interest

The authors declare that there is no conflict of interests regarding the publication of this paper. 


\section{Acknowledgment}

The authors are grateful to all the participant for their active participation and Medical officers and nursing staffs for their constant support.

\section{References}

$1 \mathrm{Xu} X-\mathrm{W}, \mathrm{Wu} \mathrm{X}-\mathrm{X}$, Jiang $\mathrm{X}-\mathrm{G}$, et al. Clinical findings in a group of patients infected with the 2019 novel coronavirus (SARS-Cov-2) outside of Wuhan, China: retrospective case series. $\mathrm{Br}$ Med J. 2020:368.

2 Menni C, Sudre CH, Steves CJ, Ourselin S, Spector TD. Quantifying additional COVID-19 symptoms will save lives. Lancet. 2020;395(10241):e107-e108.

3 Maheshkumar K, Wankhar W, Gurugubelli KR, Mahadevappa VH, Lepcha L, kumar Choudhary A. Angiotensin-converting enzyme 2 (ACE2): COVID 19 gate way to multiple organ failure syndromes. Respir Physiol Neurobiol. 2020:103548.

4 Organization WH. Coronavirus Disease ( COVID-19): Situation Report, 200. Geneva: World Health Organization; 2020:2 [online].

5 Kumar SU, Kumar DT, Christopher BP, Doss C. The rise and impact of COVID-19 in India. Front Med. 2020;7:250.

6 Maheshkumar K, Venugopal V, Poonguzhali S, Mangaiarkarasi N, Venkateswaran S, Manavalan N. Trends in the use of Yoga and Naturopathy based lifestyle clinics for the management of Non-communicable diseases (NCDs) in Tamilnadu, South India Clin Epidemiol Glob Health. 2020;8(2):647-651.

7 Chaturvedi S, Kumar N, Tillu G, Deshpande S, Patwardhan B. AYUSH, modern medicine and the Covid-19 pandemic. Indian J Med Ethics. 2020:32546457.

8 Krishnamoorthy Y, Nagarajan R, Saya GK, Menon V. Prevalence of psychological morbidities among general population, healthcare workers and COVID-19 patients amidst the COVID-19 pandemic: a systematic review and meta-analysis. Psychiatr Res. 2020;293:113382.

9 Grover S, Sahoo S, Mehra A, et al. Psychological impact of COVID-19 lockdown: an online survey from India. Indian J Psychiatr. 2020;62(4):354.

10 Snaith RP. The hospital anxiety and depression scale. Health Qual Life Outcome. 2003; $1(1): 1-4$.

11 Lee SA. Coronavirus Anxiety Scale: a brief mental health screener for COVID-19 related anxiety. Death Stud. 2020;44(7):393-401.

12 Dai L-L, Wang X, Jiang T-C, et al. Anxiety and depressive symptoms among COVID19 patients in jianghan Fangcang Shelter hospital in Wuhan, China. PloS One. 2020; 15(8), e0238416.

13 Mazza MG, De Lorenzo R, Conte C, et al. Anxiety and depression in COVID-19 survivors: role of inflammatory and clinical predictors. Brain Behav Immun. 2020;89; 594-600.

14 Vajpeyee M, Tiwari S, Jain K, et al. Yoga and music intervention to reduce depression, anxiety, and stress during COVID-19 outbreak on healthcare workers. Int J Soc Psychiatr. 2021, 00207640211006742.

15 Sahni PS, Singh K, Sharma N, Garg R. Yoga an effective strategy for self-management of stress-related problems and wellbeing during COVID19 lockdown: a crosssectional study. PloS One. 2021;16(2), e0245214.

16 James-Palmer A, Anderson E, Zucker L, Kofman Y, Daneault J-F. Yoga as an intervention for the reduction of symptoms of anxiety and depression in children and adolescents: a systematic review. Front Pediatr. 2020;8:78.

17 Gonzalez M, Pascoe M, Yang G, et al. Yoga for depression and anxiety symptoms in people with cancer: a systematic review and meta-analysis. Psycho Oncol. 2021. https://doi.org/10.1002/pon.5671. In press.

18 Venugopal V, Pamavathi R, Venkateswaran S, Gunasekaran D, Maheshkumar K. Protecting the elders from COVID-19 impact-leveraging yoga. J Fam Med Prim Care. 2020;9(8):4487. 